\title{
Xylitol Metabolism in the Isolated Perfused Rat Liver
}

\author{
By H. F. WOODS* and H. A. KREBS \\ Metabolic Research Laboratory, Nuffield Department of Clinical Medicine, \\ and Department of the Regius Professor of Medicine, Radcliffe Infirmary, \\ Oxford OX2 6HE, U.K.
}

(Received 22 December 1972)

\begin{abstract}
1. Loading the isolated perfused liver from well-fed rats with xylitol $(20 \mathrm{~mm})$ caused a depletion of adenine nucleotides and $P_{1}$ and an accumulation of $\alpha$-glycerophosphate. The ATP content fell to $66 \%$ of the control value after $10 \mathrm{~min}$ and to $32 \%$ after $80 \mathrm{~min}$. The ADP and AMP contents also fell. After $80 \mathrm{~min} 63 \%$ of the total adenine nucleotides and $59 \%$ of the $P_{1}$ had been lost. 2. The $\alpha$-glycerophosphate content rose from 0.13 to $4.74 \mu \mathrm{mol} / \mathrm{g}$ at $10 \mathrm{~min}$ and reached $8.02 \mu \mathrm{mol} / \mathrm{g}$ at $40 \mathrm{~min}$. 3. Xylitol was rapidly metabolized, the main products being glucose, lactate and pyruvate. 4. The [lactate]/ [pyruvate] ratio in the presence of xylitol rose to 30-40.5. On perfusion of livers from starved animals the main product of xylitol metabolism was glucose and the mean ratio xylitol removed/glucose formed was 1.29 (corrected for endogenous glucose and lactate production). This is close to the predicted value of 1.2. 6. Evidence is presented indicating that the loss of adenine nucleotides caused by xylitol is not due to the increased ATP consumption but to the accumulation of $\alpha$-glycerophosphate and depletion of $P_{i}$. 7. The loss of adenine nucleotides accounts for the hyperuricaemia which can occur after xylitol infusion in man. 8. The relevance of the findings to the clinical use of xylitol as an energy source is discussed.
\end{abstract}

Substances that are rapidly phosphorylated in the liver, such as fructose and glycerol, cause a depletion of hepatic ATP and also of total adenine nucleotides and of $P_{1}$ (Mäenpää et al., 1968; Raivio et al., 1969; Burch et al., 1970; Woods et al., 1970). This is due to the fact that ATP and $P_{i}$ inhibit the enzymes responsible for the irreversible breakdown of AMP (AMP deaminase and 5-nucleotidase). The present experiments were undertaken to test whether xylitol can affect hepatic nucleotides in the same way as fructose and glycerol. Xylitol is known to be rapidly converted into glucose in the liver (Ross et al., 1967; Jakob et al., 1971). This process involves phosphorylations (see Krebs \& Lund, 1966), and the rate of additional ATP consumption on addition of xylitol can be of the same order as that in the presence of fructose. The expected depletion of adenine nucleotide was in fact observed. These results are of special interest in connexion with the clinical use of xylitol as a source of energy in special situations.

\section{Materials and Methods}

Animals and diet

Female Wistar rats weighing about $200 \mathrm{~g}$ were obtained from Carworth (Europe) Ltd., Alconbury, Hunts., U.K. and were fed on a standard small-

* Present address: Medical Research Council Clinical Pharmacology Unit, Radcliffe Infirmary, Oxford OX2 6HE, U.K. animal diet (Spillers Mills Ltd., Gainsborough, Lincs., U.K.), water being provided ad libitum. This strain of rats differs in respect to several metabolic properties from that used as a routine in the Metabolic Research Laboratory. In the present strain the hepatic contents of glutamate and $\alpha$-oxoglutarate are lower, the rate of gluconeogenesis from xylitol is higher and that from alanine is lower.

\section{Reagents}

Xylitol was obtained from Sigma (London) Chemical Co. Ltd., London S.W.6, U.K., and nucleotides, coenzymes and crystalline enzymes were obtained from Boehringer Corp. (London) Ltd., London W.5, U.K.

\section{Liver perfusion}

The method of liver perfusion was that described by Hems et al. (1966). The composition of the perfusion medium has been described previously (Woods et al., 1970).

\section{Analytical methods}

Citrate was determined with citrate lyase (Gruber \& Möllering, 1966), ammonia by the method of Kirsten et al. (1963) and glutamate by the method of Bernt \& Bergmeyer (1963), as modified by Lund 
Table 1. Metabolite concentrations in the

Livers from well-fed rats were perfused with a medium containing initially 5,10 or $20 \mathrm{~mm}$-xylitol. The xylitol xylitol was present and four in the control experiments. The initial volume of the medium was $150 \mathrm{ml}$ and sampling and Methods section.

Concn. of metabolites in perfusion medium (mM)

\begin{tabular}{|c|c|c|c|c|c|c|c|c|}
\hline \multirow{2}{*}{$\begin{array}{l}\text { Time } \\
\text { (min) }\end{array}$} & \multicolumn{4}{|c|}{$5 \mathrm{~mm}-\mathrm{Xylitol}$} & \multicolumn{4}{|c|}{ 10mm-Xylitol } \\
\hline & Xylitol & Glu & $\mathrm{Lac}$ & Pyr & Xylitol & Glu & $\mathrm{La}$ & ate \\
\hline $\begin{array}{r}0 \\
15\end{array}$ & $4.73 \pm 0.01$ & $\begin{array}{l}0.2 \\
3.1\end{array}$ & & & $.28 \pm 0.21$ & & & \\
\hline 30 & $2.53 \pm 0.36$ & $4.43 \pm 0.12$ & 1.5 & $0.02 \pm 0.003$ & $5.27 \pm 0.23$ & $4.50 \pm 0.39$ & $2.23 \pm 0.12$ & 0.02 \\
\hline 45 & 二- & $5.31 \pm 0.07$ & $2.01 \pm 0.39$ & $0.03 \pm 0.01$ & - & $5.63 \pm 0.34$ & $2.52 \pm 0.27$ & $0.04 \pm 0.01$ \\
\hline 60 & $1.01 \pm 0.08$ & $5.82 \pm 0.19$ & $2.13 \pm 0.29$ & $0.12 \pm 0.04$ & $4 \pm 0$ & $6.80 \pm 0.43$ & $2.77 \pm 0.33$ & $0.05 \pm 0.01$ \\
\hline 90 & \pm 0.20 & 0.33 & \pm 0.46 & & $1.59 \pm 0.21$ & $8.95 \pm 0.42$ & $3.28 \pm 0.34$ & 0.01 \\
\hline 120 & $0.73 \pm 0.25$ & $6.75 \pm 0.36$ & $2.92 \pm 0.56$ & $0.28 \pm 0.10$ & $0.91 \pm 0.29$ & $9.64 \pm 0.50$ & $3.32 \pm 0.32$ & $0.29 \pm 0.07$ \\
\hline
\end{tabular}

(1971). Xylitol was determined by the method of West \& Rapoport (1949). Oxoglutarate was determined by the method of Bergmeyer \& Bernt (1963), malate by that of Hohorst (1963) and xylulose 5-phosphate by that of Racker (1963).

\section{Results}

Effect of xylitol concentration of the medium on the production of glucose, lactate and pyruvate by livers from well-fed rats

Xylitol was added to the perfusion medium at initial concentrations of 5,10 or $20 \mathrm{~mm}$ and the time-course of metabolic changes was followed for $120 \mathrm{~min}$. Table 1 shows the metabolite concentrations in the perfusate and Table 2 the rates of changes in $\mu \mathrm{mol} / \mathrm{min}$ per $\mathrm{g}$ of liver. Xylitol removal was rapid and the initial rates rose somewhat with the xylitol concentration from 1.55 at $5 \mathrm{~mm}$ to $1.93 \mu \mathrm{mol} / \mathrm{min}$ per $\mathrm{g}$ of liver at $20 \mathrm{~mm}$.

The initial concentration of xylitol giving halfmaximal rates of removal in perfused livers was $3.2 \mathrm{~mm}$. This value is higher than the $K_{m}$ for the NAD-linked purified cytoplasmic polyol dehydrogenase of sheep liver $(0.18 \mathrm{~mm}$ at $\mathrm{pH} 9.6$; Smith, 1962) and that of guinea-pig liver $(0.6 \mathrm{~mm}$ at $\mathrm{pH} 8.1$; Hollmann, 1969).

Glucose production was increased by xylitol over that from endogenous precursors and the final glucose concentration in the medium was greater in the xylitol perfusion than in the control perfusions. The increase was smaller than that expected on the assumption that the xylitol removed was quantitatively converted into glucose. This discrepancy was partly due to the formation of products other than glucose, i.e. lactate, and partly to an inhibition of glucose formation via glycogenolysis.
In all experiments lactate was produced throughout the perfusions, and the final lactate concentration in the medium was increased by xylitol above the control values, in which $2 \mathrm{~mm}$ was seldom exceeded. The [lactate]/[pyruvate] ratio rose in the presence of xylitol above physiological limits to $30-40$, i.e. as expected since xylitol introduces reducing equivalents (see Jakob et al., 1971).

Xylitol metabolism in livers from rats starved for $48 h$

The main product formed from xylitol in livers from starved rats was glucose; small and variable amounts of lactate were produced (Table 3 ) and pyruvate production was negligible.

The rate of xylitol removal was of the same order as in the livers of fed rats. The initial rates of glucose formation rose with increasing xylitol concentration from $1.10 \mu \mathrm{mol} / \mathrm{min}$ per $\mathrm{g}$ of liver at $5 \mathrm{~mm}^{-}$to $1.71 \mu \mathrm{mol} / \mathrm{min}$ per $\mathrm{g}$ of liver with $20 \mathrm{~mm}$-xylitol. These rates are somewhat higher than those previously measured under similar conditions $[0.67 \mu \mathrm{mol} /$ min per $\mathbf{g}$ of liver (Ross et al., 1967) and $0.65 \mu \mathrm{mol} /$ min per $\mathbf{g}$ of liver (Jakob et al., 1971)].

If glucose is the only product of xylitol metabolism the ratio (xylitol removed)/(glucose formed) should be 1.2. The values for the ratio obtained were between 1.08 and 1.25. It is uncertain whether this should be corrected for the endogenous production of glucose and lactate, because it is not known whether addition of xylitol affects the endogenous metabolism. If the correction is made the ratio has a mean value of 1.29.

Contents of intermediary metabolites in the freezeclamped perfused liver after xylitol loading

The changes in the contents of liver metabolites at different time-intervals after xylitol loading (addition 


\section{perfusion medium after xylitol loading}

concentrations in the medium are expressed as mM (mean \pm S.E.M.). The number of observations was three when decreased this by $0.5 \mathrm{ml}$ every $15 \mathrm{~min}$. The liver weights were about $7 \mathrm{~g}$. For experimental details see the Materials

Concn. of metabolites in perfusion medium (mM)

\begin{tabular}{|c|c|c|c|c|c|c|}
\hline \multicolumn{4}{|c|}{20 mm-Xylitol } & \multicolumn{3}{|c|}{ No substrate added } \\
\hline Xylitol & Glucose & Lactate & Pyruvate & Glucose & Lactate & Pyruvate \\
\hline $18.1 \pm 0.33$ & $0.28 \pm 0.05$ & $0.85 \pm 0.37$ & $0.01 \pm 0.01$ & $0.27 \pm 0.26$ & $0.26 \pm 0.02$ & $0.06 \pm 0.01$ \\
\hline - & $3.03 \pm 0.27$ & $2.12 \pm 0.09$ & $0.02 \pm 0.006$ & $2.44 \pm 0.26$ & $0.83 \pm 0.08$ & $0.13 \pm 0.05$ \\
\hline $15.1 \pm 0.25$ & $4.07 \pm 0.29$ & $2.99 \pm 0.23$ & $0.03 \pm 0.01$ & $3.44 \pm 0.27$ & $1.30 \pm 0.04$ & $0.15 \pm 0.06$ \\
\hline - & $5.13 \pm 0.47$ & $3.41 \pm 0.57$ & $0.04 \pm 0.003$ & $4.08 \pm 0.34$ & $1.59 \pm 0.06$ & $0.15 \pm 0.08$ \\
\hline $11.8 \pm 0.11$ & $6.09 \pm 0.55$ & $3.87 \pm 0.64$ & $0.04 \pm 0.01$ & $4.85 \pm 0.36$ & $1.87 \pm 0.05$ & $0.19 \pm 0.08$ \\
\hline $9.54 \pm 0.44$ & $8.61 \pm 0.74$ & $4.76 \pm 0.91$ & $0.06 \pm 0.01$ & $5.18 \pm 0.30$ & $1.91 \pm 0.16$ & $0.22 \pm 0.12$ \\
\hline $6.79 \pm 0.72$ & $11.56 \pm 0.83$ & $5.63 \pm 0.99$ & $0.06 \pm 0.02$ & $5.91 \pm 0.60$ & $2.00 \pm 0.24$ & $0.25 \pm 0.13$ \\
\hline
\end{tabular}

of 20mm-xylitol to the perfusion medium) are shown in Table 4. The main changes were, as expected, a depletion of adenine nucleotides and $P_{i}$ together with an accumulation of $\alpha$-glycerophosphate. The content of ATP fell to $66 \%$ of the control value after $10 \mathrm{~min}$ and was $32 \%$ of the control value after $80 \mathrm{~min}$. The loss of ATP was accompanied by a fall in the contents of ADP and AMP, the sum of the adenine nucleotides being $70 \%$ of the control value after $10 \mathrm{~min}$ and $37 \%$ after $80 \mathrm{~min}$. In contrast to the findings after fructose loading (Woods et al., 1970), the lost adenine nucleotides were not partly accounted for by the accumulation of IMP. There was a substantial fall in the $P_{1}$ content, from 4.25 to $1.64 \mu \mathrm{mol} / \mathrm{g}$ after $10 \mathrm{~min}$, and it then rose a little to $1.73 \mu \mathrm{mol} / \mathrm{g}$ at $80 \mathrm{~min}$.

The content of $\alpha$-glycerophosphate rose 37 -fold at $10 \mathrm{~min}$ to $4.74 \mu \mathrm{mol} / \mathrm{g}$ and further to $8.02 \mu \mathrm{mol} / \mathrm{g}$ at $40 \mathrm{~min}$. The contents of the other phosphorylated products fell but the changes were small in absolute terms. The content of xylulose 5-phosphate reached $0.18 \pm 0.01 \mu \mathrm{mol} / \mathrm{g}$ at $80 \mathrm{~min}$.

The content of some non-phosphorylated intermediates (glucose, malate, citrate and glutamate) decreased at $10 \mathrm{~min}$, probably because they were washed out from the tissue. The contents of glucose and ammonia rose to near-normal values after $80 \mathrm{~min}$.

The [fructose 6-phosphate]/[glucose 6-phosphate] ratio was of the order expected for equilibrium, and the mass-action ratio of the adenylate kinase system ([ATP][AMP]/[ADP] $]^{2}$ ) remained within the expected range at 10 and $40 \mathrm{~min}$ in spite of the large changes in the contents of the individual adenine nucleotides. The [2-phosphoglycerate]/[3-phosphoglycerate] ratio was outside the order expected for equilibrium.

A depletion of adenine nucleotides and $P_{1}$ together with $\alpha$-glycerophosphate accumulation also occurred after loading with $10 \mathrm{~mm}$-xylitol but not with $5 \mathrm{~mm}$ xylitol (Table 5).

\section{Effect of xylitol on the redox state of the hepatic $\left[N A D^{+}\right] /[N A D H]$ couple}

Xylitol shifted the redox state of the couple in the cytoplasm in the direction of reduction without affecting that of the mitochondria (Table 6). The cytoplasmic $\left[\mathrm{NAD}^{+}\right] /[\mathrm{NADH}]$ fell to about one-fifth of the normal value. Such an effect was reported by Jakob et al. (1971), but these authors also record a change in the mitochondria under similar conditions in rats starved for $24 \mathrm{~h}$. However, their initial mitochondrial redox state was abnormally low. The transient fall of the mitochondrial ratio shown in Table 6 is relatively small and of doubtful significance.

\section{Discussion}

The experiments demonstrate that xylitol, like fructose (Mäenpää et al., 1968; Woods et al., 1970) and glycerol (Burch et al., 1970; Woods \& Krebs, 1973), can cause a substantial loss of hepatic ATP, total adenine nucleotides and $P_{i}$, and a large accumulation of a phosphorylated intermediate. The concentration of ATP fell to $32 \%$, that of total adenine nucleotide to $37 \%$, that of $P_{1}$ to $40 \%$, and that of $\alpha$-glycerophosphate rose up to 60 -fold, compared with initial values. The formation of $\alpha$-glycerophosphate was greater than the loss of the phosphate in the form of $P_{i}, A T P$ and ADP, so that an uptake of phosphate by the liver from the medium must be postulated. Like fructose and glycerol, xylitol is rapidly metabolized by the liver (as already known), with glucose as the main end product. Unlike fructose, xylitol also causes a shift of the redox state of

Vol. 134 


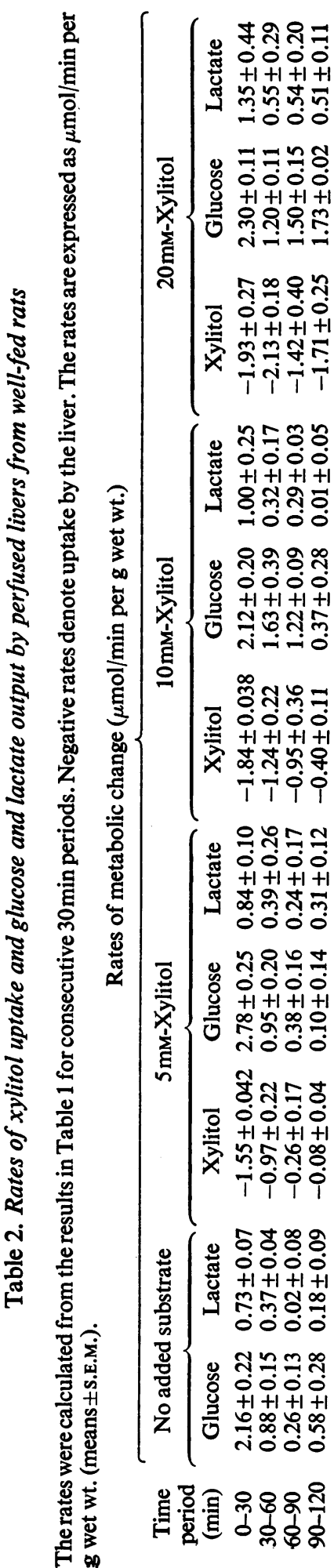

the NAD couple in the direction of reduction (see also Jakob et al., 1971).

The formation of glucose from xylitol, fructose or glycerol involves a consumption of ATP. The rate of the additional ATP consumption after loading the liver with the various precursors can be calculated on the somewhat simplifying assumptions that glucose, lactate and pyruvate are the only metabolic products, that the formation of glucose from fructose or glycerol or dihydroxyacetone requires 2 molecules of ATP/molecule of glucose, that the formation of glucose from xylitol requires 1.2 molecules of ATP/ molecule of glucose, and that the formation of lactate or pyruvate from fructose, glycerol, dihydroxyacetone or xylitol yields 1 molecule of ATP/molecule of lactate or pyruvate. The formation of the phosphorylated intermediates and their accumulation in the liver may be neglected because these are small in relation to the other metabolic products. For the purpose of the calculations it is further assumed that all the glucose formed was derived from the added substrate. This was very nearly correct in the liver of starved rats. The error in the liver of fed rats was relatively small, as at least $80 \%$ of the glucose was formed from the added precursors. The results of the calculations, based on the rates in Tables 2 and 3, and those given by Woods (1970), Woods et al. (1970) and Woods \& Krebs (1973), are shown in Table 7. The rates of additional ATP consumption are all of the same order of magnitude. There is no simple correlation between the extra ATP consumption and the loss of adenine nucleotides. Thus fructose, glycerol and xylitol cause a loss of adenine nucleotides whereas dihydroxyacetone does not. The loss is greatest with fructose, yet the rate of extra ATP consumption is higher at high xylitol concentrations and with dihydroxyacetone.

This lack of correlation becomes understandable when the rate of extra ATP consumption is compared with the total ATP turnover in the liver. The latter may be assessed from the $\mathrm{O}_{2}$ consumption (about $2.5 \mu \mathrm{mol} / \mathrm{min}$ per $\mathrm{g}$; Woods et al., 1970) and an assumed $P / O$ ratio of 3 . This gives a value of $15 \mu \mathrm{mol}$ of ATP synthesized (and utilized)/min per $\mathrm{g}$. Thus the additional ATP consumption (although it removes the whole ATP content of the tissue in about $1 \mathrm{~min}$ ) is rather less than $20 \%$ of the basal turnover.

However, there is a correlation between the accumulation of phosphorylated intermediates and the depletion of $P_{1}$ in the liver on the one hand, and the loss of adenine nucleotides on the other. Dihydroxyacetone or $5 \mathrm{~mm}$-xylitol (which in contrast to fructose, glycerol and 10 or $20 \mathrm{~mm}$-xylitol do not cause a depletion of adenine nucleotides) do not cause an accumulation of phosphorylated intermediates, though they cause an increased rate of ATP consumption comparable with that caused by fructose and glycerol. The fall of the concentration of $P_{1}$ therefore must be taken as the main factor responsible for the depletion of the 
Table 3. Effect of xylitol concentration on the rate of glucose formation in perfused livers from starved rats

Rats were starved for $48 \mathrm{~h}$. Xylitol was added after a preliminary perfusion for $38 \mathrm{~min}$ and zero time was $2 \mathrm{~min}$ later. By that time the concentration of xylitol was somewhat below the initial concentration. The rates were calculated from a plot of the metabolite concentrations in the medium against time and represent the initial rates. They are expressed as $\mu \mathrm{mol} / \mathrm{min}$ per $\mathbf{g}$ (means \pm S.E.M.). For other experimental details see the Materials and Methods section. The number of experiments is shown in parentheses.

Rates of

Initial xylitol
concentration (mM)

0
5
10

20

Glucose formation
$0.13 \pm 0.01(3)$
$1.10 \pm 0.10(3)$
$1.38 \pm 0.07(4)$
$1.71 \pm 0.07(3)$

\section{Lactate formation}

(as glucose equivalents)

$0.06 \pm 0.04$ (3)

$0.04 \pm 0.03(3)$

$0.12 \pm 0.06(4)$

$0.05 \pm 0.02(3)$
Xylitol removal

$$
\begin{aligned}
& 1.30 \pm 0.18(3) \\
& 1.50 \pm 0.14(4)
\end{aligned}
$$

$2.14 \pm 0.16(3)$
Ratio $\frac{\text { xylitol removed }}{\text { glucose formed }}$

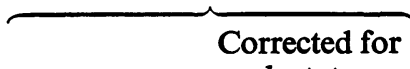

lactate

Uncorrected

production

$\overline{1.18}$
1.08
1.25

1.37

1.15

Table 4. Contents of intermediary metabolites in the perfused rat liver

Livers of fed rats were perfused with a medium containing xylitol (initial concentration $20 \mathrm{~mm}$ ) and were freezeclamped at various times after the start of perfusion. The initial values refer to livers of fed rats freeze-clamped in vivo after cervical dislocation. The results are $\mu \mathrm{mol} / \mathrm{g}$ (mean \pm S.E.M.) for three observations except for the adenine nucleotides, $\mathbf{P}_{\mathbf{i}}, \alpha$-glycerophosphate, lactate and pyruvate, where there were four observations.

Metabolite content ( $\mu \mathrm{mol} / \mathrm{g})$

\begin{tabular}{cccc}
\hline $\begin{array}{c}\text { Livers not } \\
\text { perfused }\end{array}$ & \multicolumn{3}{c}{ Livers perfused with xylitol } \\
& 10 & 40 & 80 \\
$2.22 \pm 0.07$ & $1.47 \pm 0.12$ & $0.92 \pm 0.12$ & $0.70 \pm 0.09$ \\
$0.79 \pm 0.08$ & $0.66 \pm 0.05$ & $0.47 \pm 0.03$ & $0.48 \pm 0.01$ \\
$0.26 \pm 0.05$ & $0.18 \pm 0.02$ & $0.21 \pm 0.013$ & $0.12 \pm 0.01$ \\
$3.27 \pm 0.12$ & $2.30 \pm 0.16$ & $1.61 \pm 0.12$ & $1.20 \pm 0.08$ \\
$4.25 \pm 0.25$ & $1.64 \pm 0.42$ & $1.86 \pm 0.35$ & $1.73 \pm 0.24$ \\
$0.12 \pm 0.03$ & $0.11 \pm 0.22$ & $0.008 \pm 0.01$ & $0.12 \pm 0.01$ \\
$<0.01$ & $0.04 \pm 0.003$ & $0.16 \pm 0.07$ & $0.18 \pm 0.01$ \\
$0.13 \pm 0.10$ & $4.74 \pm 1.24$ & $8.02 \pm 1.76$ & $6.99 \pm 1.19$ \\
$7.22 \pm 0.77$ & $2.42 \pm 0.23$ & $4.27 \pm 0.21$ & $7.00 \pm 0.74$ \\
$0.79 \pm 0.09$ & $0.81 \pm 0.14$ & $1.29 \pm 0.19$ & $1.57 \pm 0.24$ \\
$0.08 \pm 0.01$ & $0.02 \pm 0.0004$ & $0.017 \pm 0.0004$ & $0.02 \pm 0.01$ \\
$0.02 \pm 0.002$ & $0.01 \pm 0.002$ & $0.01 \pm 0.002$ & $0.01 \pm 0.002$ \\
$0.25 \pm 0.03$ & $0.10 \pm 0.02$ & $0.14 \pm 0.01$ & $0.16 \pm 0.02$ \\
$0.06 \pm 0.01$ & $0.02 \pm 0.01$ & $0.04 \pm 0.01$ & $0.04 \pm 0.004$ \\
$<0.01$ & $<0.01$ & $<0.01$ & $<0.01$ \\
$0.16 \pm 0.02$ & $0.02 \pm 0.01$ & $0.02 \pm 0.01$ & $<0.01$ \\
$0.04 \pm 0.01$ & $0.02 \pm 0.001$ & $0.02 \pm 0.002$ & $0.03 \pm 0.004$ \\
$0.26 \pm 0.06$ & $0.05 \pm 0.01$ & $0.06 \pm 0.002$ & $0.04 \pm 0.002$ \\
$0.006 \pm 0.0002$ & $0.003 \pm 0.0003$ & $0.004 \pm 0.0006$ & $0.004 \pm 0.0006$ \\
$0.04 \pm 0.003$ & $0.02 \pm 0.0003$ & $0.03 \pm 0.01$ & $0.03 \pm 0.002$ \\
$0.95 \pm 0.07$ & $0.44 \pm 0.23$ & $0.50 \pm 0.06$ & $0.81 \pm 0.10$ \\
$1.87 \pm 0.26$ & $1.33 \pm 0.11$ & $1.23 \pm 0.11$ & $0.86 \pm 0.13$ \\
$0.11 \pm 0.003$ & $0.11 \pm 0.01$ & $0.08 \pm 0.03$ & $0.06 \pm 0.01$ \\
$0.73 \pm 0.12$ & $0.01 \pm 0.02$ & $0.07 \pm 0.02$ & $<0.01$ \\
& & &
\end{tabular}

\section{ATP}

Time of perfusions (min) $\quad \ldots$

ADP

AMP

Total adenine nucleotides

$P_{1}$

IMP

Xylulose 5-phosphate

$\alpha$-Glycerophosphate

Glucose

Lactate

Pyruvate

Fructose 1,6-diphosphate

Glucose 6-phosphate

Fructose 6-phosphate

Glucose 1-phosphate

Phosphoenolpyruvate

2-Phosphoglycerate

3-Phosphoglycerate

Glyceraldehyde 3-phosphate

Dihydroxyacetone phosphate

Ammonia

Glutamate

$\alpha$-Oxoglutarate

Malate

Vol. 134
$1.47 \pm 0.12$

$4.74 \pm 1.24$

$0.02 \pm 0.0004$

$<0.01$

$0.02 \pm 0.01$

$0.003 \pm 0.0003$

$\pm 0.0003$

$1.33+0.11$

$0.01 \pm 0.02$
$0.70 \pm 0.09$

$1.20 \pm 0.08$

$73 \pm 0.24$

$0.18+0.01$

$1.57 \pm 0.24$

$0.02 \pm 0.01$

$0.04 \pm 0.004$

$<0.01$

$0.03 \pm 0.004$

$0.004 \pm 0.0006$

$0.03 \pm 0.002$

$0.81 \pm 0.10$

$<0.01$ 
Table 4. (Continued)

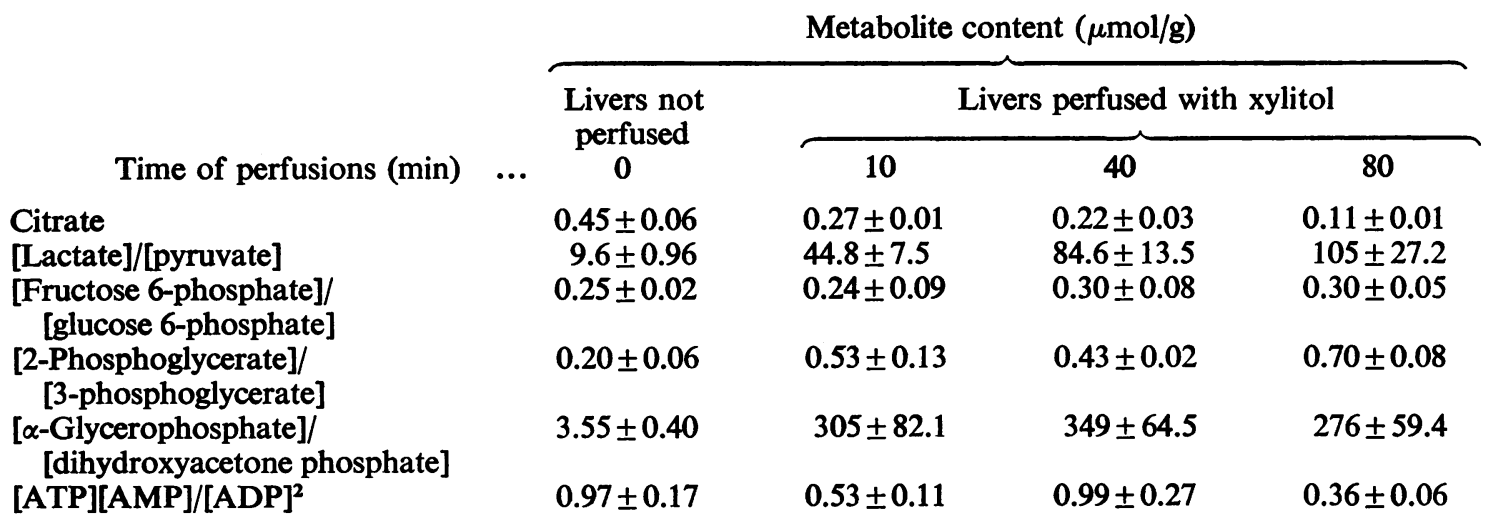

Table 5. Effect of xylitol on the metabolite contents of rat liver

The experimental conditions were as described for Table 4 except for the xylitol concentrations. The tissue was freeze-clamped $40 \mathrm{~min}$ after the start of the perfusion. Control values for livers freeze-clamped immediately after cervical dislocation and values obtained with $20 \mathrm{~mm}$-xylitol are given in Table 4.

\begin{tabular}{lllc}
\multicolumn{1}{c}{$\begin{array}{c}\text { Initial xylitol } \\
\text { concn. }\end{array}$} & $\ldots$ & $\begin{array}{c}\text { Metabolite content } \\
(\mu \mathrm{mol} / \mathrm{g} \text { wet wt. })\end{array}$ \\
\multicolumn{1}{c}{ Metabolite } & & $10 \mathrm{mM}$ \\
ATP & 2.16 & 1.48 \\
ADP & 0.47 & 0.66 \\
AMP & 0.16 & 0.21 \\
Total adenine nucleotides & 2.79 & 2.35 \\
P $_{\text {I }}$ & 4.64 & 2.34 \\
$\alpha$-Glycerophosphate & 0.86 & 3.59 \\
Xylulose 5-phosphate & 0.06 & 0.21
\end{tabular}

adenine nucleotides. It is known that a fall in the concentration of $P_{1}$ (and ATP) de-inhibits the ATPdegrading enzymes and causes a formation of IMP and adenosine (see Woods et al., 1970).

The high rates of xylitol utilization by the liver (already noted by previous investigators, see Ross et al., 1967; Jacob et al., 1971) are remarkable in view of the fact that xylitol is present in natural diets in mere traces, if at all, and that the rate of glucose formation in the course of the degradation of myoinositol and D-glucuronate is exceedingly low in rat liver (less than $0.2 \mu \mathrm{mol} / \mathrm{min}$ per $\mathrm{g}$; R. Hems, unpublished work). The high rates of xylitol utilization indicate that the enzymes converting xylitol into xylulose 5-phosphate, i.e. polyol dehydrogenase and D-xylulose kinase, as well as the enzymes of the pentose phosphate cycle, possess a high capacity in rat liver.

\section{Relevance of the findings to therapeutic uses of xylitol}

Intravenous infusion of xylitol has in recent years been recommended as a source of energy, especially during post-operative starvation periods (Baessler et al., 1962; Spitz et al., 1970; Horecker et al., 1969). According to Schumer (1971) large intravenous doses of xylitol $(4.8 \mathrm{~g} / \mathrm{h}$ per $\mathrm{kg})$ can cause abdominal pain, nausea, vomiting and rise in the serum concentrations of lactate, uric acid, bilirubin, $\mathbf{P}_{\mathbf{i}}$, glutamateoxaloacetate aminotransferase and alkaline phosphatase. The hyperuricaemia (which also occurs after fructose loading; Perheentupa \& Raivio, 1967) is in all probability a direct result of the increased degradation of the hepatic adenine nucleotides. Since the intactness of the adenine nucleotide system is a key factor in cell function, it is likely that the other abnormalities are also consequences of the loss of the adenine nucleotides. Thus intravenous infusion of relatively large amounts of xylitol is obviously not without risk.

We are indebted to Mr. R.D. Carter for competent technical assistance. This work was supported by grants from the Medical Research Council and the U.S. Public Health Service (Grant no. AM11748). 
Table 6. Effect of xylitol on the redox state of the NAD couple in perfused rat livers

The $\left[\mathrm{NAD}^{+}\right] /[\mathrm{NADH}]$ ratios were calculated as described by Williamson et al. (1967) from the values in Table 4. The tissue $\mathrm{pH}$ was taken to be 7.0.

[Free $\left.\mathrm{NAD}^{+}\right] /[$free NADH] ratio, calculated from

Material

Freeze-clamped fed liver

Liver perfused with xylitol

(initially 20mm)

$10 \mathrm{~min}$

$40 \mathrm{~min}$

$80 \mathrm{~min}$

$\overbrace{\begin{array}{c}\text { Lactate dehydrogenase } \\ \text { (cytoplasm) }\end{array}}^{\begin{array}{c}\text { Glutamate dehydrogenase } \\ \text { (mitochondrial matrix) }\end{array}}$

910

13.7

$\begin{array}{rr}225 & 9.6 \\ 140 & 8.5 \\ 114 & 14.7\end{array}$

Table 7. Hepatic ATP consumption after substrate loading

The rates of additional ATP consumption have been calculated as described in the text by using rates of metabolism derived from the results in Tables 2 and 4 and the following references: Woods (1970), Woods et al. (1970) and Woods \& Krebs (1973).

Additional ATP consumption ( $\mu \mathrm{mol} / \mathrm{min}$ per $\mathrm{g}$ wet wt.)

\begin{tabular}{lr}
\multicolumn{2}{c}{ Addition to perfusio } \\
(mM) \\
Fructose & 10 \\
Glycerol & 10 \\
Dihydroxyacetone & 10 \\
Xylitol & 5 \\
& 10 \\
& 20
\end{tabular}

$\overbrace{\text { Well-fed animal }}^{48 \mathrm{~h} \text {-starved animal }}$

1.66

2.36

3.04

1.82

2.08

1.95
2.03

1.24

2.77

1.52

1.68

2.52

\section{References}

Baessler, K. H., Prellwitz, W., Unbehaun, V. \& Long, K. (1962) Klin. Wochenschr., 40, 791-793

Bergmeyer, H. U. \& Bernt, E. (1963) in Methods of Enzymic Analysis (Bergmeyer, H. U., ed.), pp. 324-327, Academic Press, London

Bernt, E. \& Bergmeyer, H. U. (1963) in Methods of Enzymic Analysis (Bergmeyer, H. U., ed.), pp. 384-388, Academic Press, London

Burch, H. B., Lowry, O. H., Meinhardt, L., Max, P. \& Chyu, K. (1970) J. Biol. Chem. 245, 2092-2102

Gruber, W. \& Möllering, H. (1966) Biochem. Z. 346, 85-88

Hems, R., Ross, B. D., Berry, M. N. \& Krebs, H. A. (1966) Biochem. J. 101, 284-292

Hohorst, H. J. (1963) in Methods of Enzymic Analysis (Bergmeyer, H. U., ed.), pp. 328-332, Academic Press, London

Hollmann, S. (1969) in Pentoses and Pentitols (Horecker, B. L., Lang, K. \& Takagi, Y., eds.), pp. 97-108, Springer Verlag, Berlin

Horecker, B. L., Lang, K. \& Takagi, Y. (eds.) (1969) Pentoses and Pentitols, Clinical uses of pentoses and pentitols, pp. 293-400, Springer Verlag, Berlin

Jakob, A., Williamson, J. R. \& Asakura, T. (1971) J. Biol. Chem. 246, 7623-7631

Vol. 134
Kirsten, E., Gerez, C. \& Kirsten, R. (1963) Biochem. Z. 337, 312-319

Krebs, H. A. \& Lund, P. (1966) Biochem. J. 98, 210-214

Lund, P. (1971) Biochem. J. 124, 653-660

Mäenpää, P. H., Raivio, K. O. \& Kekomäki, M. P. (1968) Science 161, 1253-1254

Perheentupa, J. \& Raivio, K. O. (1967) Lancet ii, 528-531

Racker, E. (1963) in Methods of Enzymic Analysis (Bergmeyer, H. U., ed.), pp. 201-204, Academic Press, London

Raivio, K. O., Kekomäki, M. P. \& Mäenpää, P. H. (1969) Biochem. Pharmacol. 18, 2615-2624

Ross, B. D., Hems, R. \& Krebs, H. A. (1967) Biochem. J. 102, 942-951

Schumer, W. (1971) Metabolism 20, 345-347

Smith, M. G. (1962) Biochem. J. 83, 135-144

Spitz, I. M., Rubenstein, A. H., Bersohn, I. \& Baessler, K. H. (1970) Metabolism 19, 24-34

West, C. D. \& Rapoport, S. (1949) Proc. Soc. Exp. Biol. Med. (N.Y.) 70, 141-144

Williamson, D. H., Lund, P. \& Krebs, H. A. (1967) Biochem. J. 103, 514-527

Woods, H. F. (1970) D. Phil. Thesis, University of Oxford

Woods, H. F. \& Krebs, H. A. (1973) Biochem. J. 132 , 55-60

Woods, H. F., Eggleston, L. V. \& Krebs, H. A. (1970) Biochem. J. 119, 501-510 